\title{
Cosmic-Ray Diffusion and Galactic Magnetic Field Models
}

\section{Gwenael Giacinti*}

Max-Planck-Institut für Kernphysik, Postfach 103980, 69029 Heidelberg, Germany

E-mail: giacinti@mpi-hd.mpg.de

We calculate numerically the parallel and perpendicular diffusion coefficients of cosmic-rays (CR) propagating in regular and turbulent magnetic fields. For isotropic turbulence, and in the absence of any strong regular field, CRs would reside too long in the Galactic disc and overproduce secondary nuclei like boron for any reasonable value of the coherence length and strength of the turbulent field. We conclude that the propagation of Galactic CRs has to be strongly anisotropic because of a sufficiently strong regular field and, or because of an anisotropy in the turbulent field. As a consequence, the number of sources contributing to the local CR flux is found to be reduced by about two orders of magnitude compared to the case of isotropic CR diffusion.

36th International Cosmic Ray Conference -ICRC2019-

July 24th - August 1st, 2019

Madison, WI, U.S.A.

\footnotetext{
* Speaker.
} 


\section{Introduction}

The propagation of Galactic cosmic rays $(\mathrm{CR})$ is usually described by empirical diffusion models where the energy dependence of the diffusion coefficient $D(E)=D_{0}\left(E / E_{0}\right)^{\beta}$ is obtained by fitting observations [1]. In such models, discrete CR sources are approximated by a continuous distribution filling a disc with typical vertical height $h_{\mathrm{d}} \sim 0.2 \mathrm{kpc}$, while CRs diffuse in a halo of much larger height $h \sim 3-5 \mathrm{kpc}$. CRs escape in the intergalactic space when they reach the boundary of the halo. The solutions to these diffusion models are typically derived in the steadystate regime, i.e. any time-dependence in the injection history of CRs is neglected. An important constraint on these models comes from ratios of stable primaries and secondaries produced by CR interactions on gas in the Galactic disc, which depend on the grammage $X$ crossed by CRs. In particular, the boron-to-carbon $(\mathrm{B} / \mathrm{C})$ ratio measured by the AMS-02 experiment has been interpreted by the collaboration as being consistent with a $\beta \simeq 1 / 3$ power law for the energy dependence of the diffusion coefficient [2]. The normalisation $D_{0}$ is only weakly constrained using measurements of stable nuclei, since the grammage scales as $X \propto\left(h_{\mathrm{d}} / h\right) \times h^{2} / D=h_{\mathrm{d}} h / D$. Therefore, a larger value of $D_{0}$ can be compensated by an increase of the halo size $h$. This degeneracy can be broken considering the ratio of radioactive isotopes as, e.g., ${ }^{10} \mathrm{Be} /{ }^{9} \mathrm{Be}$ : Fitting successfully these ratios requires a relatively large $\mathrm{CR}$ halo, $h \simeq 5 \mathrm{kpc}$, which in turn leads to relatively large values of the normalisation constant $D_{0}=(3-8) \times 10^{28} \mathrm{~cm}^{2} / \mathrm{s}$ at $E_{0}=10 \mathrm{GeV}$ [3]. For a given magnetic field model, the trajectories of charged particles can be calculated and the diffusion coefficients can be determined numerically $[4,5,6,7,8,9]$. We noted earlier in [8] that the diffusion coefficient calculated numerically in a pure random field with $B_{\mathrm{rms}}=4 \mu \mathrm{G}$ is smaller than the one extrapolated in the diffusion picture from lower energies. In $[10,11]$, we showed that the grammage crossed by CRs in the original Jansson-Farrar Galactic magnetic field (GMF) model [12] is a factor of a few to 10 too large. The aim of the present work is to investigate these discrepancies [13]. In particular, we want to determine qualitatively those properties of a GMF model which determine if the resulting CR propagation can be reconciled with the results obtained in diffusion models. To do so, we examine several toy models which allow us to isolate the properties a GMF model should possess.

We describe our method in Sec. 2, present our results in Sec. 3, and discuss the implications of our findings for the number of sources contributing to the local CR flux in Sec. 4.

\section{Method}

The GMF consists of a regular component, ordered on kpc scales, and a turbulent field. In the following, we consider both isotropic and anisotropic turbulence. We assume power laws $\mathscr{P}(k) \propto N k^{-\alpha}$ for its power spectrum. Motivated by the consistency of the slope of the B/C ratio with Kolmogorov turbulence, we take $\alpha=5 / 3$ for the index. The fluctuations in the turbulent magnetic field extend over a large range of scales, from the dissipation scale $L_{\min } \sim 1$ AU to the outer scale $L_{\max }$, which varies from $L_{\max } \sim 10 \mathrm{pc}$ in the disc to $L_{\max } \sim 150 \mathrm{pc}$ in the halo [14]. For Kolmogorov turbulence, the coherence length $L_{\text {coh }}$ is equal to $L_{\max } / 5$. In order to cope with this large range of length scales, we construct the magnetic field in our numerical simulations on nested grids as described in Ref. [7]. This method allows us to choose the effective minimum scale $L_{\text {min }}^{\prime}$ of fluctuations in the field sufficiently small compared with the Larmor radius $R_{\mathrm{L}}=c p /(e B)$ 
of the considered CRs. For the calculation of the diffusion tensor, we propagate individual CRs in a prescribed magnetic field (regular and/or turbulent) solving the Lorentz force equation. We perform the numerical simulations with the code described in Refs. [15, 16]. Having obtained the trajectories $x_{i}^{(a)}(t)$ of $N$ CRs with energy $E$, we calculate the diffusion tensor as

$$
D_{i j}(E)=\lim _{t \rightarrow \infty} \frac{1}{2 N t} \sum_{a=1}^{N} x_{i}^{(a)} x_{j}^{(a)} .
$$

For sufficiently large times $t$, such that CRs propagate over several coherence lengths, the righthand-side becomes time-independent. Diagonalising then the tensor $D_{i j}$, it can be written as $D_{i j}=$ $\operatorname{diag}\left\{D_{\perp}, D_{\perp}, D_{\|}\right\}$where $D_{\|}$denotes the component aligned with the large-scale field.

\section{Diffusion tensor and magnetic field models}

\subsection{Pure isotropic turbulent field}

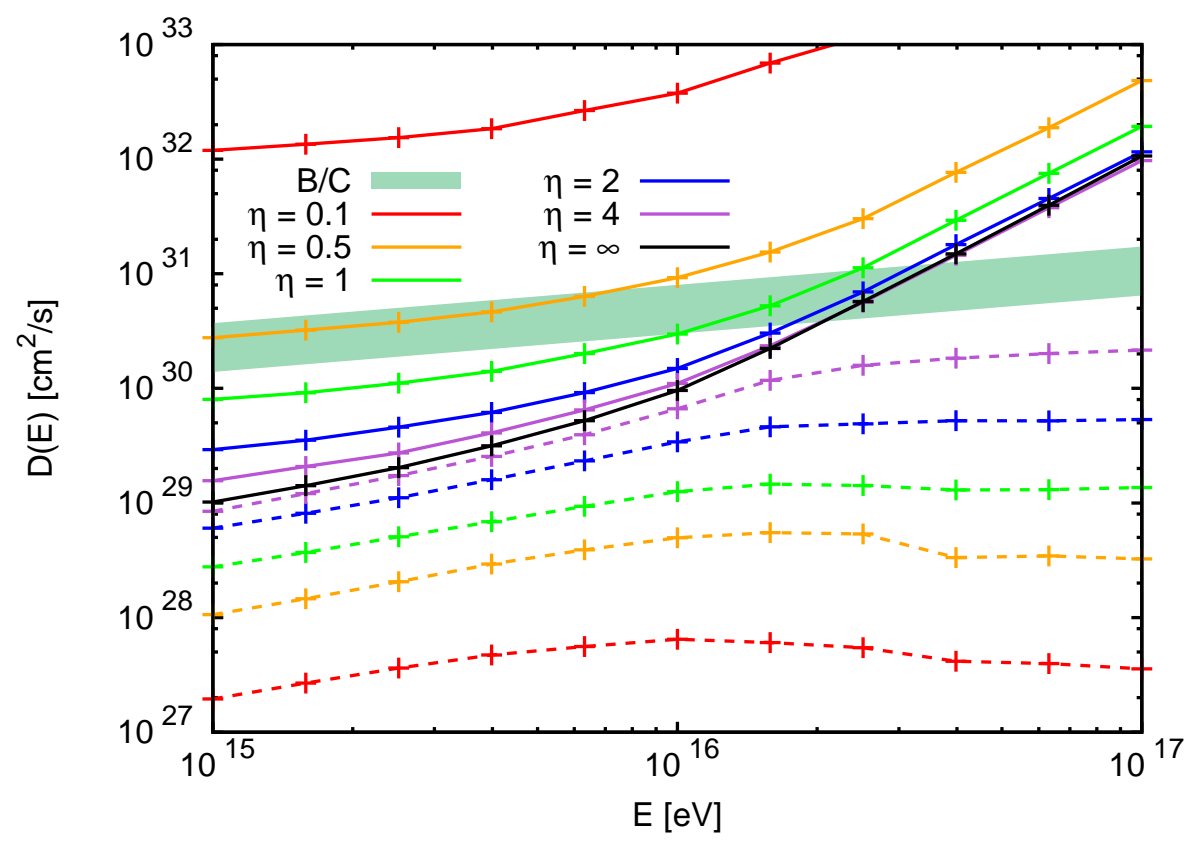

Figure 1: Parallel (solid lines) and perpendicular (dashed lines) diffusion coefficients for isotropic turbulence with a superimposed regular field. Results are presented for six levels of turbulence: $\eta=0.1,0.5$, $1,2,4$, and $\infty$ (i.e. pure turbulence). $B_{\mathrm{tot}}=\sqrt{B_{\mathrm{rms}}^{2}+B_{0}^{2}}=1 \mu \mathrm{G}$, and $L_{\mathrm{max}}=100 \mathrm{pc}$. Green area for the Kolmogorov extrapolation of $D_{0}=(3-8) \times 10^{28} \mathrm{~cm}^{2} / \mathrm{s}$ inferred at $E_{0}=10 \mathrm{GeV}$ from the B/C ratio by [1].

It is often assumed that the turbulent component of the GMF dominates over the regular one, $B_{\mathrm{rms}} \gg B_{0}$, at least in the disc. In a first approach, we set therefore the regular field $B_{0}$ to zero and consider a purely turbulent magnetic field. We plot in Fig. 1 with a black line our calculation of the diffusion coefficient in pure turbulence (level of turbulence $\eta=\infty$ ), for a total magnetic field strength set to $B_{\mathrm{tot}}=\sqrt{B_{\mathrm{rms}}^{2}+B_{0}^{2}}=1 \mu \mathrm{G}$, and for an outer scale of the turbulence equal to 
$L_{\max }=100 \mathrm{pc}$. We plot with the green area the Kolmogorov extrapolation to high energies of the value $D_{0}=(3-8) \times 10^{28} \mathrm{~cm}^{2} / \mathrm{s}$ inferred at $E_{0}=10 \mathrm{GeV}$ from the B/C ratio by [1]. The black line is below the green area by a factor of a few tens at $E \lesssim 10 \mathrm{PeV}$, which shows that the diffusion coefficient in pure isotropic turbulence is too small to satisfy the constraints from the $\mathrm{B} / \mathrm{C}$ ratio. We

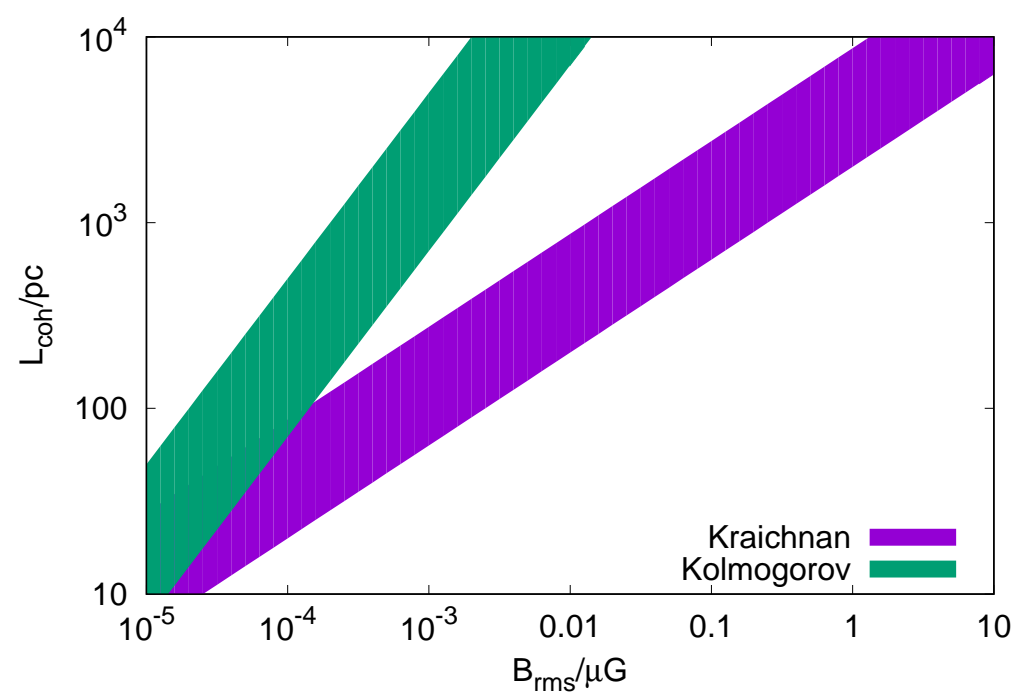

Figure 2: Allowed ranges of $B_{\mathrm{rms}}$ and $L_{\mathrm{coh}}$ compatible with $D_{0}=(3-8) \times 10^{28} \mathrm{~cm}^{2} / \mathrm{s}$ at $E_{0}=10 \mathrm{GeV}$ for Kolmogorov and Kraichnan turbulence. Typical order-of-magnitude values that are relevant for the Galactic magnetic field are: $B_{\mathrm{rms}} \sim(1-10) \mu \mathrm{G}$ and $L_{\mathrm{coh}} \lesssim \mathrm{a}$ few tens of pc.

show in Fig. 2 the ranges of magnetic field strengths, $B_{\mathrm{rms}}$, and coherence lengths, $L_{\mathrm{coh}}$, that allow the diffusion coefficient to lie in the green area, both for Kolmogorov and Kraichnan turbulence. The weak energy-dependence of $D(E) \propto E^{1 / 3}$ for Kolmogorov turbulence requires a reduction of $B_{\text {rms }}$ by a factor $\sim 1 /(100)^{3}=10^{-6}$, when keeping the coherence length $L_{\mathrm{coh}}$ fixed. Insisting instead on $B_{\mathrm{rms}} \sim$ a few $\mu \mathrm{G}$, the coherence length $L_{\mathrm{coh}}$ would have to be comparable to the size of the Galactic halo. This demonstrates that $\mathrm{CR}$ propagation cannot be described by isotropic diffusion for any reasonable value of the strength and coherence length of a predominantly turbulent isotropic field. CRs reside too long in the Galactic disc, overproducing secondary nuclei like boron. This problem can be avoided if the regular field is sufficiently strong, such that the diffusion parallel to this regular field becomes considerably faster than in the isotropic diffusion picture.

\subsection{Isotropic turbulence with a uniform regular field}

We now add a uniform magnetic field directed along the $z$ direction to the isotropic turbulent field. As a result, the propagation of CRs becomes anisotropic, leading to a diffusion tensor with $D_{i j}=\operatorname{diag}\left\{D_{\perp}, D_{\perp}, D_{\|}\right\}$and $D_{\|}>D_{\perp}$. Fig. 1 shows $D_{\|}$(solid lines) and $D_{\perp}$ (dashed lines) for six values of the level of turbulence $\eta=B_{\mathrm{rms}} / B_{0}: \eta=0.1$ (red lines), 0.5 (orange), 1 (green), 2 (blue), 4 (purple), and $\infty$. The limit $\eta \rightarrow \infty$ corresponds to isotropic turbulence, and thence isotropic diffusion. We set $B_{\mathrm{tot}}=\sqrt{B_{\mathrm{rms}}^{2}+B_{0}^{2}}=1 \mu \mathrm{G}$, and $L_{\max }=100 \mathrm{pc}$. Decreasing $\eta$, the difference between $D_{\|}$and $D_{\perp}$ increases, while keeping the order $D_{\|}>D_{\infty}>D_{\perp}$ intact, where 
$D_{\infty}(E)$ denotes the diffusion coefficient for pure isotropic turbulence. One can see that cases with $\eta \geq 1$ all give too small parallel and perpendicular diffusion coefficients to accommodate for the value required by the $\mathrm{B} / \mathrm{C}$ ratio. More generally, the perpendicular diffusion coefficient is too small for any value of $\eta$, as expected because of $D_{\perp}<D_{\infty}$. We note, as previously pointed out by $[6,17]$, that the slope of $D_{\perp}$ is larger than $1 / 3$. Assessing whether this slope continues down to $\mathrm{GeV}$ energies or not is difficult due to the limited energy range one can probe. If it does, this would only make perpendicular diffusion even less relevant for the escape of CRs from the Galaxy.

On the contrary, the parallel diffusion coefficients for cases with a strong regular magnetic field $(\eta<1)$ are able to fulfill the constraints from the B/C ratio. In Fig. 1, the solid orange line for $\eta=0.5$ is at the required level, assuming Kolmogorov turbulence and $B_{\text {tot }}=1 \mu \mathrm{G}$. A slightly lower value of $\eta$ would be preferred for $B_{\mathrm{tot}} \gtrsim 4 \mu \mathrm{G}$. This good match between the diffusion coefficient required from the $\mathrm{B} / \mathrm{C}$ ratio and $D_{\|}$for $\eta=0.5$ assumes however that the regular field in the halo is perpendicular to the Galactic plane. In reality, this field is more likely to be at an angle from the plane, such as e.g. for the "X-field" in the GMF model of Ref. [12]. If so, CRs have to travel longer distances along the regular field before escape, and therefore lower values of $\eta$ would be required. Let us note that if the regular magnetic field were to be purely toroidal, then CR escape would be controlled by $D_{\perp}$, which would be problematic. However, the field is purely toroidal in none of the existing regular GMF models, since they all have a non-zero pitch angle. In these models, the distance along lines of the regular field within the Galaxy is usually no more than a few tens of kiloparsecs. All the results presented in this Section can be rescaled using the fact that the grammage traversed by CRs scales roughly as $\propto\left(l_{\mathrm{d}} / l\right) \times l^{2} / D_{\|}=l_{\mathrm{d}} l / D_{\|}$, where $l$ is the total distance along the regular field before escape, and $l_{\mathrm{d}}$ the distance along the regular field inside the Galactic disc $(|z| \leq 200 \mathrm{pc})$, i.e. the region where most of the grammage is accumulated.

We showed in Refs. $[10,11]$ that one can reproduce the correct grammage CRs cross in the GMF model of Jansson and Farrar [12] if one reduces the turbulent field by a factor $\sim 8-10$. CRs then propagate mainly along the regular field and, since the field in this model contains a $z$ component, CRs propagate efficiently towards the Galactic halo.

\subsection{Anisotropic turbulent field}

Another possible solution to the discrepancy between estimates of the CR diffusion coefficient from the $\mathrm{B} / \mathrm{C}$ ratio and the calculations presented for pure isotropic turbulence is that interstellar turbulent magnetic fields may be anisotropic. Assuming a non-zero regular field along $z$, we generate anisotropic turbulence using the same grid as in the previous Sections, but rescale its components as: $B_{z} \rightarrow \sqrt{\xi} B_{z}$, and $B_{x, y} \rightarrow \sqrt{(3-\xi) / 2} B_{x, y}$, with $0<\xi<3$. The value of $B_{\text {rms }}$ remains unchanged from that of the initial grid, while the component of the turbulent field in the direction of the regular field can be either enhanced or decreased. In Fig. 3, we present calculations of the parallel (solid lines) and perpendicular (dashed lines) diffusion coefficients, for $\eta=0.5, L_{\max }=100 \mathrm{pc}$, and $B_{\mathrm{tot}}=\sqrt{B_{\mathrm{rms}}^{2}+B_{0}^{2}}=1 \mu \mathrm{G}$ (left panel) or $4 \mu \mathrm{G}$ (right panel). Five levels of anisotropy $\xi$ are shown, see the keys. For reference, the case of isotropic turbulence $(\xi=1)$ is shown with the black lines. The green area corresponds to the Kolmogorov extrapolation of the diffusion coefficient inferred by [1] from the $\mathrm{B} / \mathrm{C}$ ratio at $E_{0}=10 \mathrm{GeV}$. The value of the perpendicular diffusion coefficient does not vary significantly with $\xi$, while the variations in the parallel diffusion coeffi- 

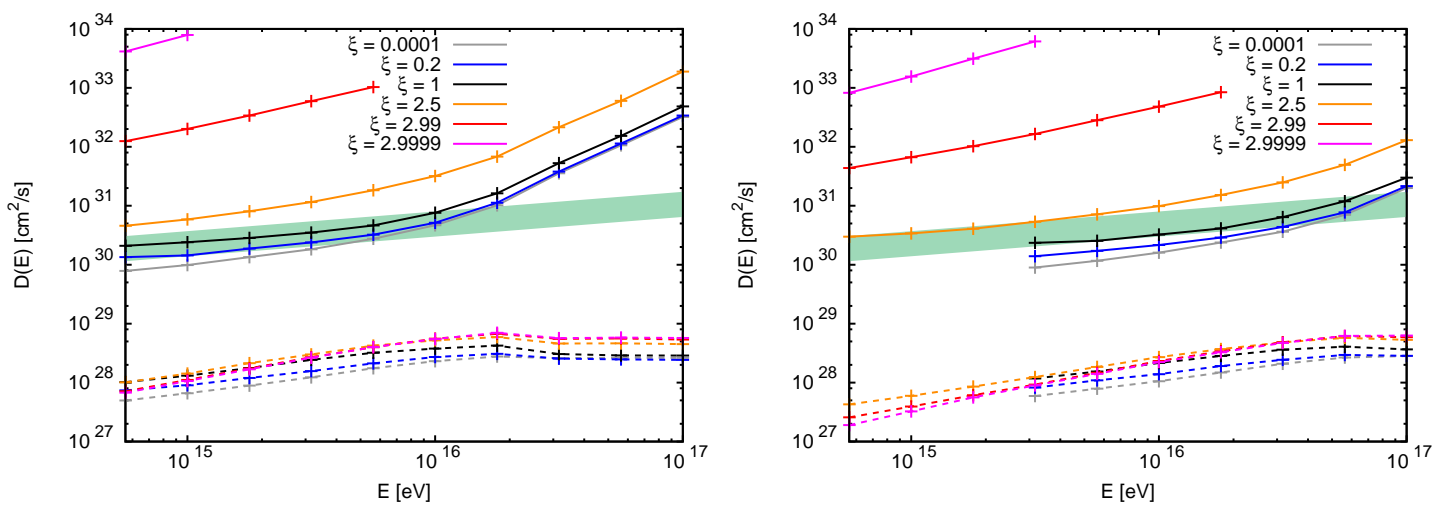

Figure 3: Parallel (solid lines) and perpendicular (dashed lines) diffusion coefficients for anisotropic turbulence with $\xi=0.0001,0.2,2.5,2.99,2.9999$, and with a superimposed regular field satisfying $\eta=0.5$. $L_{\max }=100 \mathrm{pc}$, and $B_{\mathrm{tot}}=1 \mu \mathrm{G}$ (left panel) or $4 \mu \mathrm{G}$ (right panel). Black line for isotropic turbulence $(\xi=1)$.

cient can be substantially larger for some values of $\xi$. For the two cases where the turbulence is enhanced in the directions perpendicular to the regular field $(\xi=0.0001$ and $\xi=0.2$ ), both the parallel and perpendicular diffusion coefficients are reduced by a small factor in comparison with isotropic turbulence. On the contrary, for $\xi>1$, the parallel diffusion coefficient increases in comparison with isotropic turbulence. From the right panel, one can deduce that the normalisation of the diffusion coefficient at low energies for $\xi=2.5$ is larger than that for $\xi=1$ by a factor $\approx 2.7$. Such a value is however still small compared with the increase one would need in order to bring the cases with $\eta \geq 2$ into the green area, even though $\xi=2.5$ already corresponds to a non-negligible anisotropy. For $\xi=2.99, D_{\|}$starts to be an order of magnitude larger than for $\xi=2.5$, see solid red lines in Fig. 3. The solid magenta lines for $\xi=2.9999$ show that if nearly all the power of the turbulence is along $B_{z}$, then the diffusion coefficient would increase by orders of magnitude. Therefore anisotropic turbulence can alleviate the constraints put on $\eta$ in Sect. 3.2, by allowing for larger turbulence levels $\eta$ to satisfy the constraints from the B/C ratio. However, a very large level of anisotropy in $k$-space would be needed for the difference to be substantial.

\section{Number of sources contributing to the local CR flux}

In order to illustrate the impact of anisotropic diffusion on the assumptions underlying Galactic CR physics, we consider as an example the number of sources contributing to the local CR flux. More specifically, we discuss inspired by Refs. [18] the question if a single 2-3 Myr source can dominate the $\mathrm{CR}$ flux in the $10 \mathrm{TeV}$ energy range. Let us assume that the source injects instantaneously $10^{50} \mathrm{erg}$ in CRs with the power law $Q(E)=Q_{0}\left(E / E_{0}\right)^{-\alpha}$ and $\alpha \simeq 2.2$. At a given energy, the functional behaviour of the observed CR flux from a single source at the distance $L$ and the age $t$ can be divided into three regimes: For $2 D t \lesssim L^{2}$, the diffuse flux is exponentially suppressed, while for intermediate times it is

$$
I(E) \simeq \frac{c}{4 \pi} \frac{Q(E)}{V(t)}
$$


with $V(t)=\pi^{3 / 2} D_{\perp} D_{\|}^{1 / 2} t^{3 / 2}$. When the diffusion front reaches the edge of the Galactic CR halo with size $h$, CRs start to escape. Thus in the third, final time regime, the slope of the CR intensity steepens as $I(E) \propto E^{-\alpha-1 / 3}$ in the case of Kolmogorov turbulence. Note that for all estimates of the type $2 D t \sim L^{2}$ or $2 D t \sim h^{2}$, the relevant component of the diffusion tensor should be used.

We consider first the standard case of isotropic diffusion. Then at the reference energy $E_{*}=$ $10 \mathrm{TeV}$, the isotropic diffusion coefficient equals $D\left(E_{*}\right) \sim 5 \times 10^{29} \mathrm{~cm}^{2} / \mathrm{s}$, and the size of the diffusion front is $L(t)=\sqrt{2 D t} \simeq 2.5 \mathrm{kpc}$ for $t=2 \mathrm{Myr}$. Assuming a Galactic CR halo size of $\sim 5 \mathrm{kpc}$, we can use Eq. (4.1) to estimate the contribution of this single source to the observed CR intensity as $E_{*}^{2.8} I\left(E_{*}\right) \simeq 200 \mathrm{GeV}^{1.8} \mathrm{sr}^{-1} \mathrm{~s}^{-1} \mathrm{~m}^{-2}$. This corresponds only to $1 / 100$ of the observed CR intensity at this energy. Therefore one expects that a large number of sources contributes to the local CR intensity in the case of isotropic diffusion. As a result, features of individual sources like a varying nuclear composition or maximal energies are washed out, and both the primary and secondary CR fluxes should be smooth. Moving on to the case of anisotropic diffusion, we expect from Fig. 1 that $D_{\|} \simeq 200 D_{\perp} \simeq D_{\text {iso }}$ for a regular field perpendicular to the disc, where $D_{\text {iso }}$ denotes the isotropic diffusion coefficient satisfying the $\mathrm{B} / \mathrm{C}$ constraints. If the regular field has only a small $z$ component, as in the case of the JF model, the required ratio $D_{\|} / D_{\perp}$ would be even larger. Thus the volume occupied by CRs shrinks by a factor $\gtrsim 200$, and a single source can contribute a fraction of order $\mathscr{O}(1)$ to the total CR intensity. Consequently, one expects breaks in the CR primary fluxes as a result of the varying composition and spectral shape of different sources, resulting in step-like features in the secondary ratios as discussed in [18].

\section{Conclusions}

We calculated the diffusion coefficients of charged CRs, propagating them in regular and turbulent magnetic fields by solving the Lorentz equation. We showed that CRs reside too long in the Galactic disc if the magnetic field is dominated by an isotropic turbulent component for any reasonable combination of field strengths and coherence lengths. As a result, CRs scattering on gas in the Galactic disc overproduce secondary nuclei like boron. Therefore propagation of Galactic CRs has to be strongly anisotropic, because of a sufficiently strong regular field, and/or an anisotropy of the turbulent field. A viable solution in our toy model is given by $D_{\|} \simeq 200 D_{\perp} \simeq D_{\text {iso }}$ for the diffusion coefficients. As a consequence, the volume that CRs from a single source occupy at intermediate times is reduced by a factor $\mathscr{O}(100)$ compared to the case of isotropic CR diffusion. Similarly, the number of sources contributing to the local CR flux is reduced and, therefore, single sources can dominate the $\mathrm{CR}$ flux already in the $\mathrm{TeV}$ energy range.

\section{References}

[1] A. W. Strong, I. V. Moskalenko, V. S. Ptuskin, Cosmic-ray propagation and interactions in the Galaxy, Ann. Rev. Nucl. Part. Sci. 57 (2007) 285 [astro-ph/ 0701517 ].

[2] M. Aguilar et al. [AMS Collaboration], Precision Measurement of the Boron to Carbon Flux Ratio in Cosmic Rays from 1.9 GV to 2.6 TV with the Alpha Magnetic Spectrometer on the International Space Station, Phys. Rev. Lett. 117 (2016) 231102. 
[3] V. S. Ptuskin, A. Soutoul, Astron. Astrophys. 337 (1998) 859; G. Jóhannesson et al., Bayesian analysis of cosmic-ray propagation: evidence against homogeneous diffusion, ApJ 824 (2016) 16 [arXiv: 1602 . 02243]; C. Evoli, D. Gaggero, D. Grasso, L. Maccione, Cosmic-Ray Nuclei, Antiprotons and Gamma-rays in the Galaxy: a New Diffusion Model, JCAP 0810 (2008) 018, Erratum: JCAP 1604 (2016) E01 [arXiv: 0807 .4730].

[4] F. Casse, M. Lemoine, G. Pelletier, Transport of cosmic rays in chaotic magnetic fields, Phys. Rev. D 65 (2002) 023002 [astro-ph/ 0109223 ].

[5] E. Parizot, GZK horizon and magnetic fields, Nucl. Phys. Proc. Suppl. 136 (2004) 169 [astro-ph/0409191].

[6] D. De Marco, P. Blasi, T. Stanev, Numerical propagation of high energy cosmic rays in the Galaxy. I. Technical issues, JCAP 0706 (2007) 027 [arXiv: 0705 . 1972].

[7] G. Giacinti, M. Kachelrieß, D. V. Semikoz, G. Sigl, Cosmic Ray Anisotropy as Signature for the Transition from Galactic to Extragalactic Cosmic Rays, JCAP 1207 (2012) 031 [arXiv:1112.5599].

[8] G. Giacinti, M. Kachelrieß, D. V. Semikoz, Filamentary Diffusion of Cosmic Rays on Small Scales, Phys. Rev. Lett. 108 (2012) 261101 [arXiv: 1204 .1271].

[9] G. Giacinti, M. Kachelrieß, D. V. Semikoz, Anisotropic Cosmic Ray Diffusion and its Implications for Gamma-Ray Astronomy, Phys. Rev. D 88 (2013) 023010 [arXiv:1306.3209].

[10] G. Giacinti, M. Kachelrieß, D. V. Semikoz, Explaining the Spectra of Cosmic Ray Groups above the Knee by Escape from the Galaxy, Phys. Rev. D 90 (2014) R041302 [arXiv: 1403.3380 ].

[11] G. Giacinti, M. Kachelrieß, D. V. Semikoz, Escape model for Galactic cosmic rays and an early extragalactic transition, Phys. Rev. D 91 (2015) 083009 [arXiv: 1502.01608 ].

[12] R. Jansson, G. R. Farrar, A New Model of the Galactic Magnetic Field, ApJ 757 (2012) 14 [arXiv: 1204.3662$]$; The Galactic Magnetic Field, ApJ 761 (2012) L11 [arXiv:1210. 7820].

[13] G. Giacinti, M. Kachelriess, D. V. Semikoz, Reconciling cosmic ray diffusion with Galactic magnetic field models, JCAP 1807 (2018) 051 [arXiv: 1710 . 08205].

[14] M. Iacobelli, M. Haverkorn, E. Orru, R. F. Pizzo, J. Anderson, R. Beck, M. R. Bell, A. Bonafede, Studying Galactic interstellar turbulence through fluctuations in synchrotron emission: First LOFAR Galactic foreground detection, Astron. Astrophys. 558 (2013) A72 [arXiv:1308.2804].

[15] G. Giacinti, M. Kachelrieß, D. V. Semikoz, G. Sigl, Ultrahigh Energy Nuclei in the Galactic Magnetic Field, JCAP 1008 (2010) 036 [arXiv: 1006.5416 ].

[16] G. Giacinti, M. Kachelrieß, D. V. Semikoz, G. Sigl, Ultrahigh Energy Nuclei in the Turbulent Galactic Magnetic Field, Astropart. Phys. 35 (2011) 192 [arXiv:1104.1141].

[17] A. P. Snodin, A. Shukurov, G. R. Sarson, P. J. Bushby, L. F. S. Rodrigues, Global diffusion of cosmic rays in random magnetic fields, MNRAS 457 (2016) 3975 [arXiv: 1509.03766 ].

[18] M. Kachelrieß, A. Neronov, D. V. Semikoz, Signatures of a two million year old supernova in the spectra of CR protons, antiprotons and positrons, Phys. Rev. Lett. 115 (2015) 181103 [arXiv:1504.06472]; CR signatures of a 2-3 Myr old local supernova, [arXiv:1710.02321]. 